1 Analysis of SARS-CoV-2-controlled autophagy reveals spermidine, MK-2206, and niclosamide as

2 putative antiviral therapeutics

3

\title{
4 Authors/Affiliation
}

5 Nils C. Gassen ${ }^{1 \$}$, Jan Papies ${ }^{2,3}$, Thomas Bajaj ${ }^{1}$, Frederik Dethloff ${ }^{4}$, Jackson Emanuel ${ }^{2,3}$, Katja Weckmann ${ }^{1}$, 6 Daniel E. Heinz ${ }^{1}$, Nicolas Heinemann ${ }^{2,3}$, Martina Lennarz ${ }^{1}$, Anja Richter ${ }^{2,3}$, Daniela Niemeyer ${ }^{2,3}$, Victor M. Corman ${ }^{2,3}$, Patrick Giavalisco ${ }^{4}$, Christian Drosten ${ }^{2,3}$, Marcel A. Müller $2,3,5 \$$

$9 \quad{ }^{1}$ Department of Psychiatry and Psychotherapy, University of Bonn, 53127 Bonn, Germany

10 Institute of Virology, Charité-Universitätsmedizin Berlin, corporate member of Freie Universität 11 Berlin, Humboldt-Universität zu Berlin, and Berlin Institute of Health, 10117 Berlin, Germany

$12{ }^{3}$ German Centre for Infection Research (DZIF), partner site Charité, 10117 Berlin, Germany

$13 \quad{ }^{4}$ Max Planck Institute for Aging, 50931 Cologne, Germany

$14{ }^{5}$ Martsinovsky Institute of Medical Parasitology, Tropical and Vector Borne Diseases, Sechenov 15 University, 119991 Moscow, Russia

16

17 Contact: ${ }^{\$}$ Correspondence should be addressed to Nils C. Gassen (nils.gassen@ukbonn.de) or Marcel 18 A. Müller (marcel.mueller@charite.de)

19 


\section{Abstract}

22 Severe acute respiratory syndrome coronavirus 2 (SARS-CoV-2) poses an acute threat to public health

23 and the world economy, especially because no approved specific drugs or vaccines are available.

24 Pharmacological modulation of metabolism-dependent cellular pathways such as autophagy reduced propagation of highly pathogenic Middle East respiratory syndrome (MERS)-CoV. Here we show that SARS-CoV-2 infection limits autophagy by interfering with multiple metabolic pathways and that compound-driven interventions aimed at autophagy induction reduce SARS-CoV-2 propagation in vitro. In-depth analyses of autophagy signaling and metabolomics indicate that SARSCoV-2 reduces glycolysis and protein translation by limiting activation of AMP-protein activated kinase (AMPK) and mammalian target of rapamycin complex 1 (mTORC1). Infection also downregulates autophagy-inducing spermidine, and facilitates AKT1/SKP2-dependent degradation of autophagyinitiating Beclin-1 (BECN1). Targeting of these pathways by exogenous administration of spermidine, AKT inhibitor MK-2206, and the Beclin-1 stabilizing, antihelminthic drug niclosamide inhibited SARSCoV-2 propagation by 85,88 , and $>99 \%$, respectively. In sum, SARS-CoV-2 infection causally diminishes autophagy. A clinically approved and well-tolerated autophagy-inducing compound shows potential 
The current pandemic of severe acute respiratory syndrome coronavirus 2 (SARS-CoV-2) poses an imminent threat to global health. As of 15 April 2020, 1,878,489 individuals were infected in $>200$ countries, with $>119,000$ fatalities (1). SARS-CoV-2 infections cause CoV-associated disease 19 (COVID19), which can lead to severe atypical pneumonia in humans (2). Currently, there are no approved therapeutics or vaccines available. The development and licensing of new FDA-approved drugs takes years, which is problematic given the urgent need for effective therapies against novel, rapidly emergent diseases like COVID-19. Antiviral drug screenings are commonly based on testing FDAapproved compound libraries against cellular and viral components (3). However, these undirected approaches lack functional insights into how the drugs affect virus propagation. Risk evaluations for drug repurposing and development of new therapeutics would benefit from rational drug design founded on known SARS-CoV-2-host interactions.

Compound-based targeting of cellular proteins that are essential for the virus life cycle has led to the discovery of broadly reactive drugs against a range of CoVs (3-6). As virus propagation strongly depends on energy and catabolic substrates of host cells, drug target identification should consider the metabolism of infected cells (3). Autophagy, a highly conserved cytosolic degradation process of long-lived proteins, lipids, and organelles in eukaryotic cells, is tightly controlled by metabolism $(7,8)$. During autophagy, intracellular macromolecules are recycled by incorporation into LC3B-lipidated autophagosomes (AP) and degradation into their monomers, such as fatty and amino acids, after fusion with low pH lysosomes (9). In the case of highly pathogenic Middle East respiratory syndrome (MERS)-CoV, we recently showed that autophagy is limited by a virus-induced AKT1-dependent activation of the E3-ligase S-phase kinase-associated protein 2 (SKP2), which targets the key autophagy initiating protein Beclin-1 (BECN1) for proteasomal degradation (10). Congruently, inhibition of SKP2 by different compounds, including clinically approved drugs, stabilized BECN1 and limited MERS-CoV propagation, indicating that autophagy-inducing compounds hold promise for evaluation as antiviral drugs. This paper investigates the impact of SARS-CoV-2 infection on cell metabolism and the downstream effects on autophagy, thereby identifying multiple targets for the application of approved drugs and the development of new antiviral therapies.

We aimed to characterize the effect of SARS-CoV-2 infection on autophagy by applying previously established assays (10) and additionally including detailed analyses of upstream autophagy regulators according to expert-curated guidelines for detecting autophagy (11). First, we explored whether SARSCoV-2 reduces autophagic flux, a measure of autophagic degradation activity. We infected human bronchial epithelial cells NCl-H1299 and monkey kidney cells (VeroFM) with SARS-CoV-2 strain Munich (multiplicity of infection (MOI) of 0.0005 ) or heat-inactivated virus (mock-infected cells) and tested autophagic flux by co-treatment with bafilomycin A1 (BafA1). BafA1 is a specific inhibitor of vacuolar $\mathrm{H}^{+}$-ATPase interfering with lysosome acidification and degradation of AP cargo and preventing the 
fusion of APs with lysosomes to autophagy-active autophagolysosomes (AL) (11). Based on our previous experience (10), we chose a low $\mathrm{MOI}$ of 0.0005 and time points 8,24 , and 48 hours post infection to monitor autophagy during the exponential growth of SARS-CoV-2 reaching maximum titers of $10^{7}$ genome equivalents per $\mathrm{ml}(\mathrm{GE} / \mathrm{ml} ; \mathrm{NCl}-\mathrm{H} 1299)$ and $10^{10} \mathrm{GE} / \mathrm{ml}$ (VeroFM) at 48 hours post infection (Figure S1a). We found that $100 \mathrm{nM}$ of BafA1 was sufficient to induce maximal LC3B lipidation levels in both cell cultures (Figure S1b-d). Immunoblotting of LC3B-II/I in the presence of $100 \mathrm{nM}$ BafA1 indicated that mock-infected compared to SARS-CoV-2-infected cells showed a significant increase of LC3B-II over LC3B-I due to the BafA1-induced inhibition of autophagic flux (Figures 1a-b). At 8 hours post infection, LC3B-II levels were slightly elevated in SARS-CoV-2-infected cells but could still be enhanced by BafA1 treatment. At 24 and 48 hours, (vehicle- or) BafA1-treated and SARS-CoV-2 infected cells showed equally limited autophagic flux (Figures 1a-b). A reduction of autophagic flux was further corroborated by elevated levels of the autophagy receptor SQSTM1/P62 in SARS-CoV-2infected cells (Figures 1c-d). Furthermore, we used a well-established autophagy reporter plasmid, ptfLC3, to assess lysosomal-autophagosomal fusion, a hallmark of autophagic flux (12). The number of ALs (in red) in mRFP and EGFP dual-tagged LC3B-expressing and SARS-CoV-2 infected NCI-H1299 cells was clearly reduced compared to mock-infected cells (Figures 1e-f), indicating virus-induced reduction of AP and AL fusion. Quantification showed that the total number of ALs was reduced from a mean of 88 to 62 vesicles per cell, whereas the number of APs per cell was comparable (Mock = 56 vesicles/cell; SARS-CoV-2 $=51$ vesicles/cell). The ratio of APs to ALs shifted in SARS-CoV-2-infected cells, indicating strong AP accumulation (Mock=0.707 \pm 0.053 ; SARS-CoV-2 $=0.921 \pm 0.058$ ) (Figure 1e). Taken together, SARS-CoV-2 strongly reduced the autophagic flux in both cell lines, in a fashion similar to MERS-CoV (10).

The initiation of autophagy is a cumulative result of multiple protein and signaling cascades that are involved in the energy and nutrient sensing of cells $(7,11)$. The AMP- activated protein kinase (AMPK) and the mammalian target of rapamycin complex 1 (mTORC1) kinase are in continuous crosstalk with glucose and protein homeostasis respectively $(13,14)$. We monitored key proteins of the AMPK and mTORC1 pathways and their regulation/degradation by Western blot analyses after infection with SARS-COV-2 (Figure 2). SARS-CoV-2 infection influenced most of the analyzed components of the AMPK/mTORC1 pathway. Phosphorylated, active forms of AMPK, AMPK substrates (LXRXX), AMPK downstream targets (TSC2 and ULK1), and mTORC1 were downregulated upon SARS-CoV-2 infection, suggesting a virus-induced reduction of cellular glycolysis, protein translation, and cell growth. In addition, we found increased levels of phosphorylated AKT1, which activates the negative regulator of BECN1, SKP2 (10). Low BECN1 levels and subsequently reduced ATG14 phosphorylation and oligomerization (Figure 2, lower left panel) explain the lack of fusion of APs with lysosomes observed in Figure 1e-f as ATG14 oligomers facilitate fusion via SNARE-proteins, SNAP29 and STX17 (10). In 
summary, SARS-CoV-2 alters autophagy-relevant signaling and at the same time appears to hamper AP/lysosome fusion efficiency via autophagy.

111 To further explore the direct impact of SARS-CoV-2 infection on AMPK/mTORC1, we performed a 112 metabolomics profiling analysis in VeroFM cells 24 hours post infection using an elevated MOI of 0.1 113 to guarantee ubiquitous infection of cells (Figure 3). The metabolite profiles were analyzed by 114 multivariate principal component analysis (PCA), an unsupervised statistical method suitable for analyzing and classifying metabolomics datasets (15). The profiles clearly separated into SARS-CoV-2 and control group (Figure S2a). Altogether, the levels of 25 metabolites were significantly altered by SARS-CoV-2 infection (Supplementary Table 1). We performed pathway analyses in order to gain insights into the cellular and mechanistic consequences of a SARS-CoV-2 infection. We retrieved eight affected pathways using the significantly altered metabolites from Supplementary Table 1 (Figure 3a).

120 Glutathione (GSH) metabolism, pyrimidine metabolism, and aminoacyl-tRNA biosynthesis had the 121 most pronounced changes. Metabolites of the butanoate metabolism as well as the alanine, aspartate 122 and glutamate metabolism were also elevated. In contrast, the tricarboxylic acid (TCA) cycle, glutamine 123 and glutamate metabolism, and glyoxylate and dicarboxylate metabolism were reduced by SARS-CoV124 2. The volcano plot visualizes the metabolites with large magnitude fold changes. We identified seven 125 altered metabolites: Cys-glycine, N-acetylputrescine, adenosine, putrescine, homocysteine and 126 cysteine levels were elevated whereas fructose-6P was strongly depleted (Figure 3b). Putative links 127 between the different pathways during SARS-CoV-2 infection are shown in Figure 3c. We observed 128 elevated ATP levels that might facilitate energy-dependent processes such as virus replication and 129 entry (Figure 3c, blue). High lactic acid and low fructose-6P levels indicate exhaustive glycolysis activity 130 (Figure 3c, green). Decreased levels of TCA metabolites (Figure 3c, purple) further support a switch 131 from (mitochondria-dependent) oxidative phosphorylation to lactic acid fermentation, to provide 132 NAD+ for glycolytic ATP production. Consequently, we observed a reduced AMP/ATP ratio (Figure 3c, 133 blue) limiting AMPK activation (16) and subsequently autophagy (see Figure 2). Limited autophagy 134 might prevent degradation of viral products and might enhance the availability of non-autophagic 135 membrane material to generate double-membrane vesicles that are generated during coronavirus 136 replication $(17,18)$. Interestingly, despite reduction of protein-degrading autophagy, we observed 137 strong upregulation of amino acids, especially those linked to glutathione metabolism, which is 138 responsible for elimination of reactive oxygen species (ROS). This might be essential to limit the 139 mitochondrion-derived ROS that are generated during excessive ATP production (19) and should be 140 investigated in future studies. Further investigations should also clarify why the observed upregulated 141 amino acid levels do not activate mTORC1 (see Figure 2, pmTOR $52448 / \mathrm{mTOR}^{5}$ and pULK1 ${ }^{5757} /$ ULK1). 142 However, spatially distinct pools of mTORC1 proteins might serve as an explanation (20). 
143 Interestingly, putrescine (Figure 3c, red) was increased whereas its downstream products of the 144 polyamine biosynthesis pathway, spermidine and spermine, were strongly reduced. The putrescine 145 increase might be explained by a SARS-CoV-2-induced inhibition of spermidine synthase, the enzyme 146 that synthesizes spermidine from putrescine (Figure 3c, red). Spermidine is an important polyamine 147 capable of inducing autophagy (21) and responsible for hypusination of the eukaryotic translation 148 initiation factor elF5A (22). elF5A activates the autophagy transcription factor TFEB and regulates 149 proteins responsible for mitochondrial respiration (23).

150 Next, we targeted different components of the metabolic/autophagic pathway by exogenous 151 administration of selective inhibitors, approved drugs, or cell metabolites, and explored the effect on 152 SARS-CoV-2 propagation (Figure 4). Cell viability tests were performed for inhibitors and drugs to 153 exclude toxicity (Figure S3a). VeroFM cells were infected with an MOI of 0.0005 and treated 1 hour 154 post infection with different concentrations of each substance according to the manual instructions or 155 previous publications. Virus growth was monitored by real-time RT-PCR in cell culture supernatants 24 156 and 48 hours post infection (Figure S3b-d). DFMO $(500 \mu \mathrm{M})$ blocks ornithine-to-putrescine synthesis 157 (24) and was previously described as an RNA virus inhibitor upon pretreatment (25) but had minor 158 effects on SARS-CoV-2 growth upon post-treatment, whereas spermidine (100 $\mu \mathrm{M})$ and spermine (100 $159 \mu \mathrm{M}$ ) inhibited SARS-CoV-2 propagation by up to 66\% (Figure 4a, upper left panel, Figure S3b,c). AICAR $160(25 \mu \mathrm{M})$, a known AMPK activator (26), slightly induced virus growth at 24 hours post infection (Figure 161 4a, upper right panel), which seems contradictory, as AMPK is downregulated upon SARS-CoV-2 162 infection (see Figure 2), but could be explained by AMPK-induced mTORC1 inhibition or AMPK163 unrelated functions of AICAR (27). Alternatively, AICAR-dependent AMPK activation might be 164 insufficient to restore autophagic flux (see Figure 1). In addition, mTORC1 inhibition by rapamycin (0.3 $\mu \mathrm{M}$ ) clearly induced virus growth (Figure 4a, right panel, Figure S3d,e). Rapamycin, which has previously been proposed as a treatment option for COVID-19 (28), possibly enhances the inhibitory effects of SARS-CoV-2 on mTORC1 (see Figure 2) to reduce cellular protein translation, which would serve as an additional explanation for the above-mentioned elevated amino acid levels (see Figure $\mathbf{3 c}$, orange). These results suggest that detailed molecular and functional analyses should be performed 170 for rapamycin before considering its use in clinical trials. The AKT1 inhibitor MK-2206 (1 $\mu \mathrm{M})$, which is 171 currently being tested in a clinical phase 2 study against breast cancer (29), reduced SARS-CoV-2 172 propagation up to 50\% (Figure 4a, lower right, Figure S3d,e). AKT1 blocks mTORC1 inhibitor TSC2 (30) 173 and further supports the suggestion that up-regulation of mTORC1 components has antiviral effects. 174 As AKT1 inhibition results in BECN1 up-regulation and autophagy induction (10, 31), SARS-CoV-2 175 growth inhibition was expected. Direct blocking of the negative BECN1 regulator SPK2 by previously 176 described inhibitors SMIP004, SMIP004-7, valinomycin, and niclosamide (10) showed SARS-CoV-2 177 growth inhibition from 50 (SMIP004, SMIP004-7) to over $99 \%$ in case of valinomycin and niclosamide 
178 (Figure 4a, lower panel, Figure S3d,e). We further confirmed that the dominant intervention of 179 niclosamide during SARS-CoV-2 infection acts on autophagy induction, as adding BafA1 after 180 niclosamide treatment showed an enhancing effect on the lipidation of LC3B as reflected by comparable LC3B-II/I ratios between mock- and SARS-CoV-2-infected cells (Figure 4b). However, we cannot exclude that the activity of niclosamide as a hydrogen ionophore has additional inhibitory functions, e.g. by blocking endosomal acidification (32), which is important for SARS-CoV-2 entry (6). We further used our in vitro model to explore the possibility of prophylactic treatment with spermidine and niclosamide, which are well-tolerated, clinically applied (33) or FDA-approved (34) compounds, respectively. To assess the efficacy of pretreatment, VeroFM cells were preincubated with spermidine $(100 \mu \mathrm{M})$ or niclosamide $(5 \mu \mathrm{M})$ for 24 hours. Cells were infected with SARS-CoV-2 using an $\mathrm{MOI}$ of 0.05 without further compound treatment and virus growth was monitored in supernatants for 24 hours (Figure 4c, Figure S3f). In both cases, SARS-CoV-2 growth was reduced by $70 \%$, suggesting that both compounds exhibit long-lasting antiviral effects, and supporting further investigation into prophylactic use of these compounds. For clinical use, half-maximal inhibitory concentration (IC50) of compounds should be in a non-toxic range and reach adequate plasma levels (35). The IC50 was 149 $\mu \mathrm{M}\left(\mathrm{R}^{2}=0.71\right)$ for spermidine, $0.09 \mu \mathrm{M}\left(\mathrm{R}^{2}=0.95\right)$ for $\mathrm{MK}-2206$, and $0.17 \mu \mathrm{M}\left(\mathrm{R}^{2}=0.63\right)$ for niclosamide, based on plaque-forming infectious units (Figure 4c). Maximal inhibition of infectious virus at nontoxic concentrations was identified at $333.3 \mu \mathrm{M}$ for spermidine (85\%), at 3.7 $\mu \mathrm{M}$ for MK-2206 (88\%), and at $1.24 \mu \mathrm{M}$ for niclosamide ( $>99 \%)$. Whereas plasma levels and pharmacokinetics of spermidine are yet to be evaluated, peak plasma concentrations were $0,176 \mu \mathrm{g} / \mathrm{ml}(0.43 \mu \mathrm{M})$ for MK-2206 (36) and 0.25 to $6.0 \mu \mathrm{g} / \mathrm{ml}(0.76-18.35 \mu \mathrm{M})$ for niclosamide (37), encouraging use in clinical trials. In summary, our data show that highly pathogenic SARS-CoV-2 reprograms the metabolism of cells and limits AMPK/mTORC1 activation and autophagy. Our mechanistic approach, including metabolism, protein/phosphoprotein analyses and targeted SARS-CoV-2 inhibition assays, identified multiple cellular targets for the development of new and application of already available antiviral compounds.

\section{Acknowledgments}

We thank Patricia Tscheak and Antje Richter (Charité) for excellent technical assistance and Terry Jones for editing of the manuscript. Funding: CD was supported by BMBF-RAPID 01KI1723A. Author contributions: N.C.G. and M.A.M. designed and conceived the work. N.C.G., J.P., T.B., F.D., J.E., N.H., D.E.H., M.L., A.R., M.A.M. carried out experiments. N.C.G., J.P., T.B., J E., K.W. V.M.C., D.N., P.G., C.D., M.A.M. analyzed data or contributed essential material. N.C.G., M.A.M. wrote the main paper text. N.C.G., T.B. prepared all figures. All authors reviewed the paper. Competing interests: None of the authors declares a competing interest. 


\section{References}

214 1. WHO, https://www.who.int/emergencies/diseases/novel-coronavirus-2019. accessed 15 April 2020, (2020).

2. W. J. Guan et al., Clinical Characteristics of Coronavirus Disease 2019 in China. N Engl J Med, (2020).

3. J. Kindrachuk et al., Antiviral potential of ERK/MAPK and PI3K/AKT/mTOR signaling modulation for Middle East respiratory syndrome coronavirus infection as identified by temporal kinome analysis. Antimicrob Agents Chemother 59, 1088-1099 (2015).

4. S. Pfefferle et al., The SARS-coronavirus-host interactome: identification of cyclophilins as target for pan-coronavirus inhibitors. PLoS Pathog 7, e1002331 (2011).

5. A. Lundin et al., Targeting membrane-bound viral RNA synthesis reveals potent inhibition of diverse coronaviruses including the middle East respiratory syndrome virus. PLoS Pathog 10, e1004166 (2014).

6. M. Hoffmann et al., SARS-CoV-2 Cell Entry Depends on ACE2 and TMPRSS2 and Is Blocked by a Clinically Proven Protease Inhibitor. Cell, (2020).

7. Y. Ohsumi, Historical landmarks of autophagy research. Cell Res 24, 9-23 (2014).

8. K. Weckmann et al., Metabolomics profiling reveals differential adaptation of major energy metabolism pathways associated with autophagy upon oxygen and glucose reduction. Sci Rep 8, 2337 (2018).

9. T. Kirisako et al., The reversible modification regulates the membrane-binding state of Apg8/Aut7 essential for autophagy and the cytoplasm to vacuole targeting pathway. $J$ Cell Biol 151, 263-276 (2000).

10. N. C. Gassen et al., SKP2 attenuates autophagy through Beclin1-ubiquitination and its inhibition reduces MERS-Coronavirus infection. Nat Commun 10, 5770 (2019).

11. D. J. Klionsky et al., Guidelines for the use and interpretation of assays for monitoring autophagy (3rd edition). Autophagy 12, 1-222 (2016).

12. S. Kimura, T. Noda, T. Yoshimori, Dissection of the autophagosome maturation process by a novel reporter protein, tandem fluorescent-tagged LC3. Autophagy 3, 452-460 (2007).

13. I. Tamargo-Gomez, G. Marino, AMPK: Regulation of Metabolic Dynamics in the Context of Autophagy. Int J Mol Sci 19, (2018).

14. K. Inoki, J. Kim, K. L. Guan, AMPK and mTOR in cellular energy homeostasis and drug targets. Annu Rev Pharmacol Toxicol 52, 381-400 (2012).

15. B. Worley, R. Powers, Multivariate Analysis in Metabolomics. Curr Metabolomics 1, 92107 (2013).

16. D. G. Hardie, AMPK--sensing energy while talking to other signaling pathways. Cell Metab 20, 939-952 (2014).

17. K. Knoops et al., SARS-coronavirus replication is supported by a reticulovesicular network of modified endoplasmic reticulum. PLoS Biol 6, e226 (2008).

18. Y. Choi, J. W. Bowman, J. U. Jung, Autophagy during viral infection - a double-edged sword. Nat Rev Microbiol 16, 341-354 (2018).

19. M. P. Murphy, How mitochondria produce reactive oxygen species. Biochem J 417, 113 (2009).

20. R. Hatakeyama et al., Spatially Distinct Pools of TORC1 Balance Protein Homeostasis. Mol Cell 73, 325-338 e328 (2019).

21. T. Eisenberg et al., Induction of autophagy by spermidine promotes longevity. Nat Cell Biol 11, 1305-1314 (2009). 
22. D. J. Puleston et al., Polyamines and elF5A Hypusination Modulate Mitochondrial Respiration and Macrophage Activation. Cell Metab 30, 352-363 e358 (2019).

23. H. Zhang et al., Polyamines Control elF5A Hypusination, TFEB Translation, and Autophagy to Reverse B Cell Senescence. Mol Cell 76, 110-125 e119 (2019).

24. H. M. Wallace, A. V. Fraser, Inhibitors of polyamine metabolism: review article. Amino Acids 26, 353-365 (2004).

25. B. C. Mounce et al., Inhibition of Polyamine Biosynthesis Is a Broad-Spectrum Strategy against RNA Viruses. J Virol 90, 9683-9692 (2016).

26. J. E. Sullivan et al., Inhibition of lipolysis and lipogenesis in isolated rat adipocytes with AICAR, a cell-permeable activator of AMP-activated protein kinase. FEBS Lett 353, 3336 (1994).

27. J. Kirchner, B. Brune, D. Namgaladze, AICAR inhibits NFkappaB DNA binding independently of AMPK to attenuate LPS-triggered inflammatory responses in human macrophages. Sci Rep 8, 7801 (2018).

28. Y. Zhou et al., Network-based drug repurposing for novel coronavirus 2019nCoV/SARS-CoV-2. Cell Discov 6, 14 (2020).

29. Y. Xing et al., Phase II trial of AKT inhibitor MK-2206 in patients with advanced breast cancer who have tumors with PIK3CA or AKT mutations, and/or PTEN loss/PTEN mutation. Breast Cancer Res 21, 78 (2019).

30. K. Inoki, Y. Li, T. Zhu, J. Wu, K. L. Guan, TSC2 is phosphorylated and inhibited by Akt and suppresses mTOR signalling. Nat Cell Biol 4, 648-657 (2002).

31. R. C. Wang et al., Akt-mediated regulation of autophagy and tumorigenesis through Beclin 1 phosphorylation. Science 338, 956-959 (2012).

32. A. Jurgeit et al., Niclosamide is a proton carrier and targets acidic endosomes with broad antiviral effects. PLoS Pathog 8, e1002976 (2012).

33. M. Wirth et al., The effect of spermidine on memory performance in older adults at risk for dementia: A randomized controlled trial. Cortex 109, 181-188 (2018).

34. FDA, https://www.accessdata.fda.gov/scripts/cder/daf/index.cfm?event=overview.proces s\&AppINo=018669. (accessed 01 April 2020).

35. C. Schwarz et al., Safety and tolerability of spermidine supplementation in mice and older adults with subjective cognitive decline. Aging (Albany NY) 10, 19-33 (2018).

36. D. H. Ahn et al., Results of an abbreviated phase-Il study with the Akt Inhibitor MK2206 in Patients with Advanced Biliary Cancer. Sci Rep 5, 12122 (2015).

37. W. E. Jones, Niclosamide as a treatment for Hymenolepis diminuta and Dipylidium caninum infection in man. Am J Trop Med Hyg 28, 300-302 (1979). 
bioRxiv preprint doi: https://doi.org/10.1101/2020.04.15.997254; this version posted April 15, 2020. The copyright holder for this preprint

(which was not certified by peer review) is the author/funder. All rights reserved. No reuse allowed without permission.

\section{Figure 1}

299

a
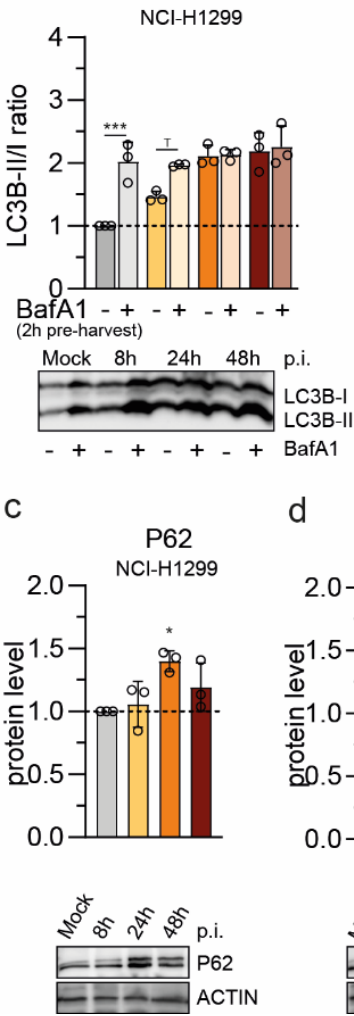

b

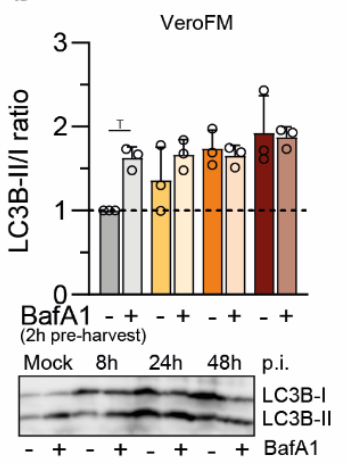

e f LC3B-mRFP-EGFP
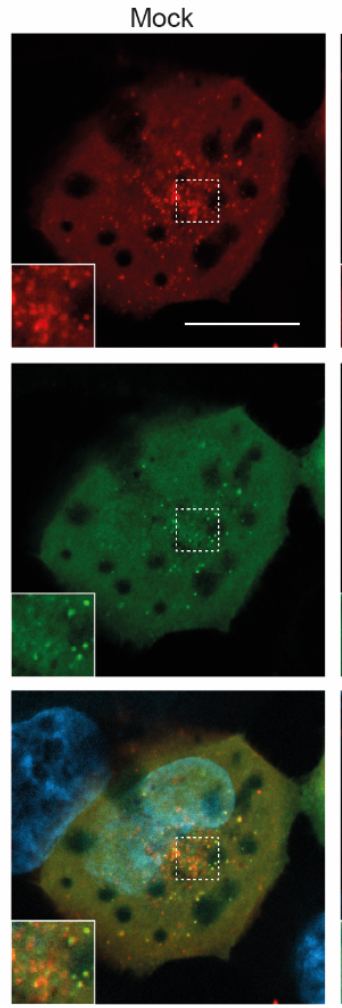

SARS-CoV-2
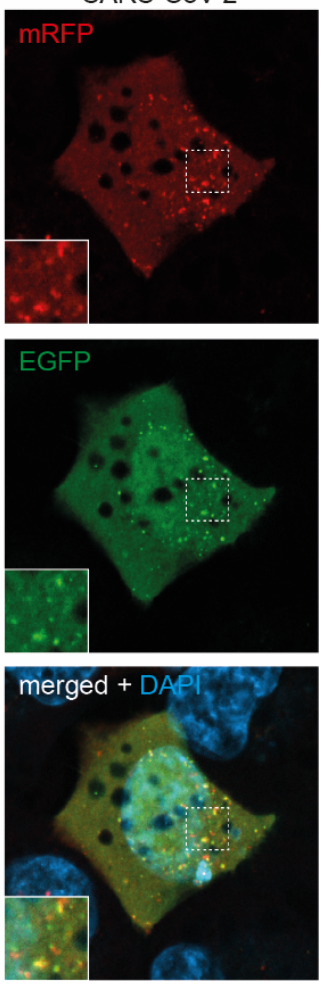

d
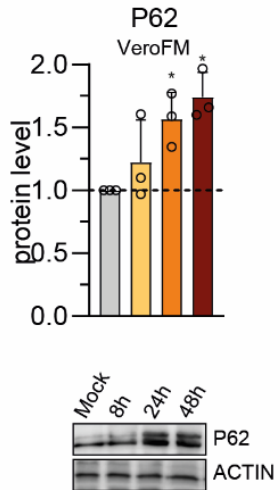

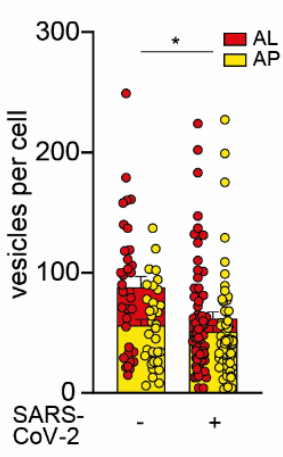

Fig.1: SARS-CoV-2 decreases lysosomal-autophagosomal fusion. (a,b) SARS-CoV-2 blocks autophagic flux. NCI-H1299 cells (a) and VeroFM cells $(\mathbf{b})$ were infected with SARS-CoV-2 $(\mathrm{MOI}=0.0005)$ and incubated with bafilomycin A1 (BafA1) or vehicle (DMSO) for $2 \mathrm{~h}$ before samples were harvested at $8 \mathrm{~h}, 24 \mathrm{~h}$, or $48 \mathrm{~h}$ post infection (h p.i.). The ratios of LC3B-II/I were determined by Western blotting. (c-d) SARS-CoV-2 stabilizes autophagy receptor P62 protein levels. Cells were infected analogously to (a-b) and harvested at indicated time points p.i. without additional treatment. P62 protein levels were determined by Western blotting. (e,f) SARS-CoV-2 blocks fusion of autophagosomes (AP) with lysosomes. $\mathrm{NCl}-\mathrm{H} 1299$ cells were transfected with tandem fluorescent-tagged LC3B (mRFP and EGFP) and infected with SARS-CoV-2 (MOI $=0.0005$ ). Twenty-four hours later, cells were fixed and analyzed by fluorescence microscopy. Vesicles with both green and red fluorescence (APs) and with red fluorescence only (autolysosomes, AL) were counted. In all panels error bars denote standard error of mean derived from $n=3$ biologically independent experiments. $T p<0.1, * p<0.05, * * * p<0.001$ (two-way ANOVA in a,b, one-way ANOVA in c,d, t-test in e. Abbreviations: LC3B, microtubule-associated protein $1 \mathrm{~A} / 1 \mathrm{~B}$ light chain 3B; mRFP, monomeric red fluorescent protein; EGFP, enhanced green fluorescent protein. 


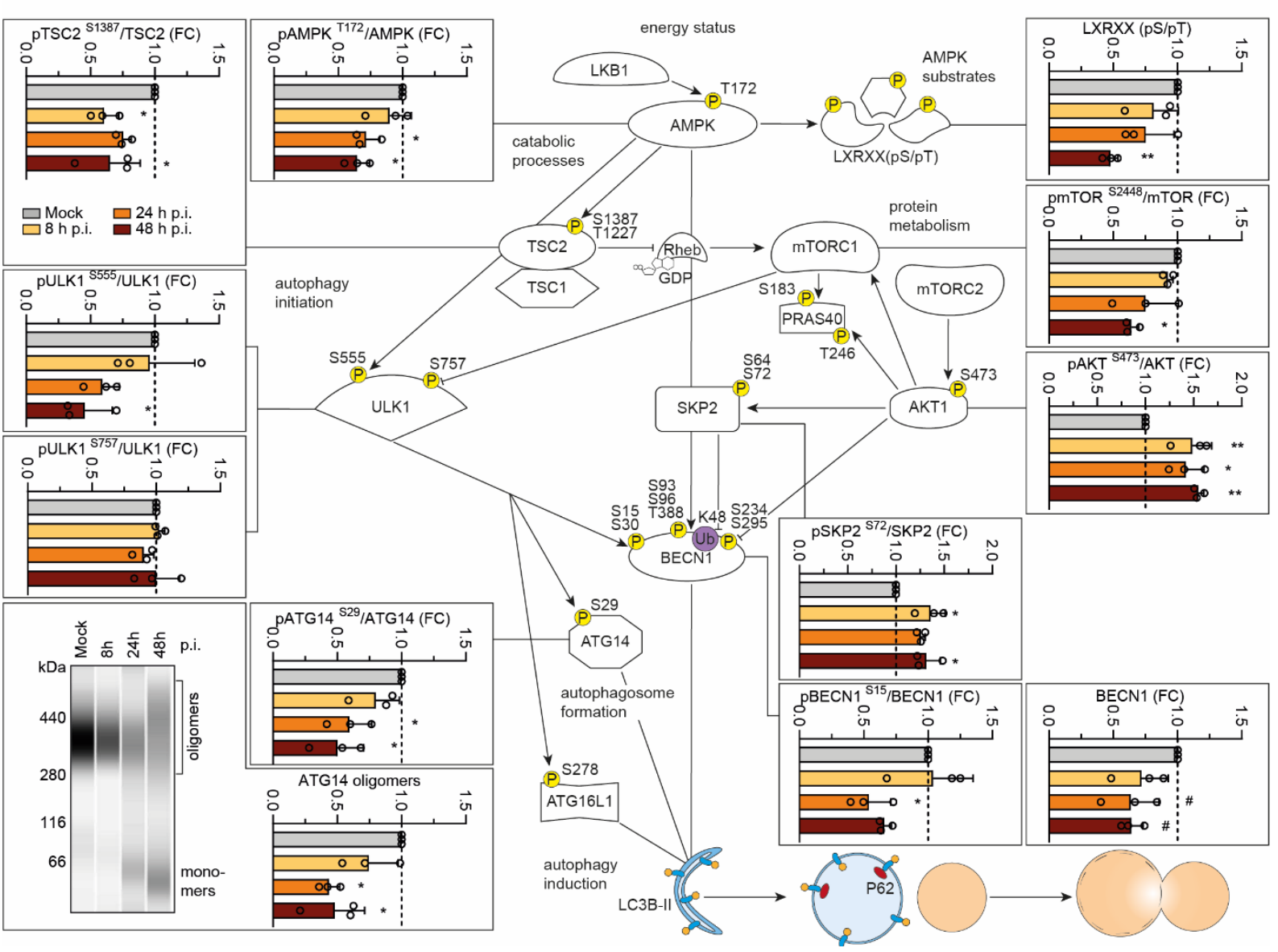

Fig.2: SARS-CoV-2 hijacks autophagy signaling on multiple regulatory levels. VeroFM cells were infected with SARS-CoV-2 $(\mathrm{MOI}=0.0005)$ and harvested at $8 \mathrm{~h}, 24 \mathrm{~h}$, or $48 \mathrm{~h}$ post infection ( $\mathrm{h}$ p.i.). Protein levels and phosphorylation status of selected autophagy-relevant proteins were determined by Western blotting. For analysis of ATG14 oligomers (bottom panel, left) cells were incubated with a chemical crosslinker (DSS) $2 \mathrm{~h}$ prior to cell harvest (for detailed protocol see methods section). Cell extracts were separated and immunoblotted using Wes (ProteinSimple) capillary electrophoresis. Error bars in all panels denote standard error of mean derived from $n=3$ biologically independent experiments. $T p<0.1, * p<0.05, * * * p<0.001$ (one-way ANOVA; Bonferroni correction (post hoc)). Abbreviations: AMPK, AMP-activated protein kinase; TSC1/2, tuberous sclerosis 1/2; mTORC1/2, mammalian target of rapamycin complex 1/2; PRAS40, proline-rich AKT1 substrate 1; SKP2, S-phase kinase-associated protein 2; BECN1, Beclin-1; ATG, autophagy-related; Rheb, Ras homolog enriched in brain; ULK1, Unc-51like kinase 1; 


\section{Figure 3}
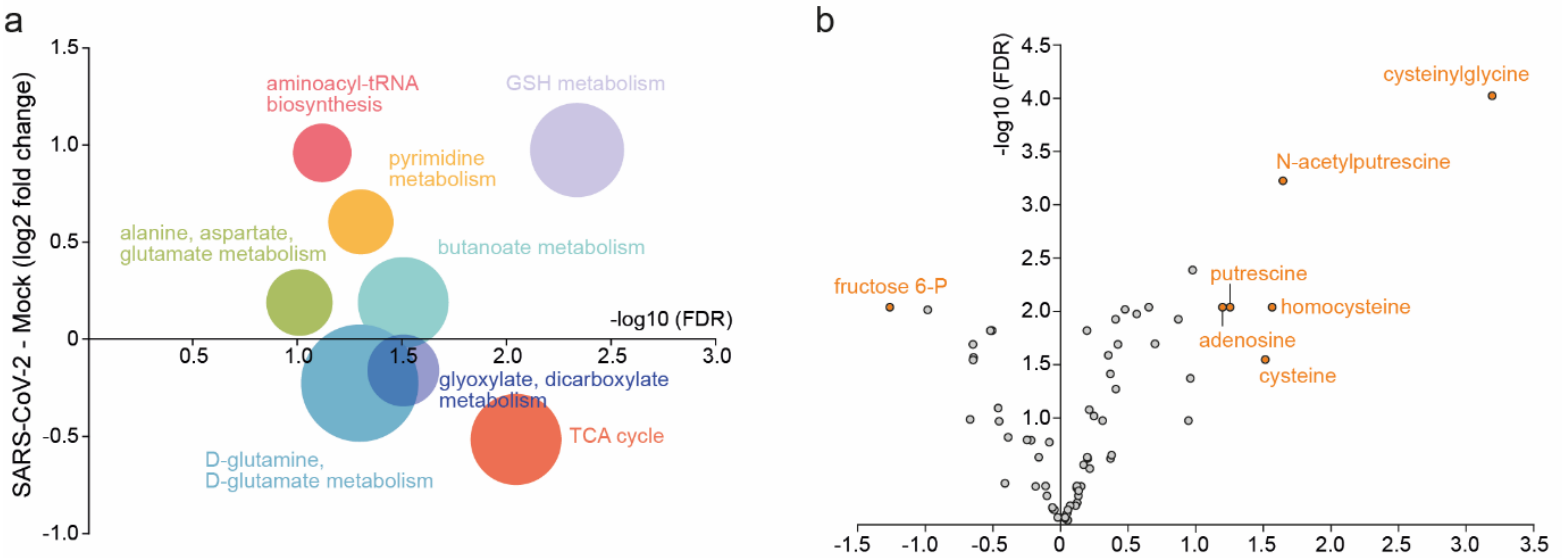

C

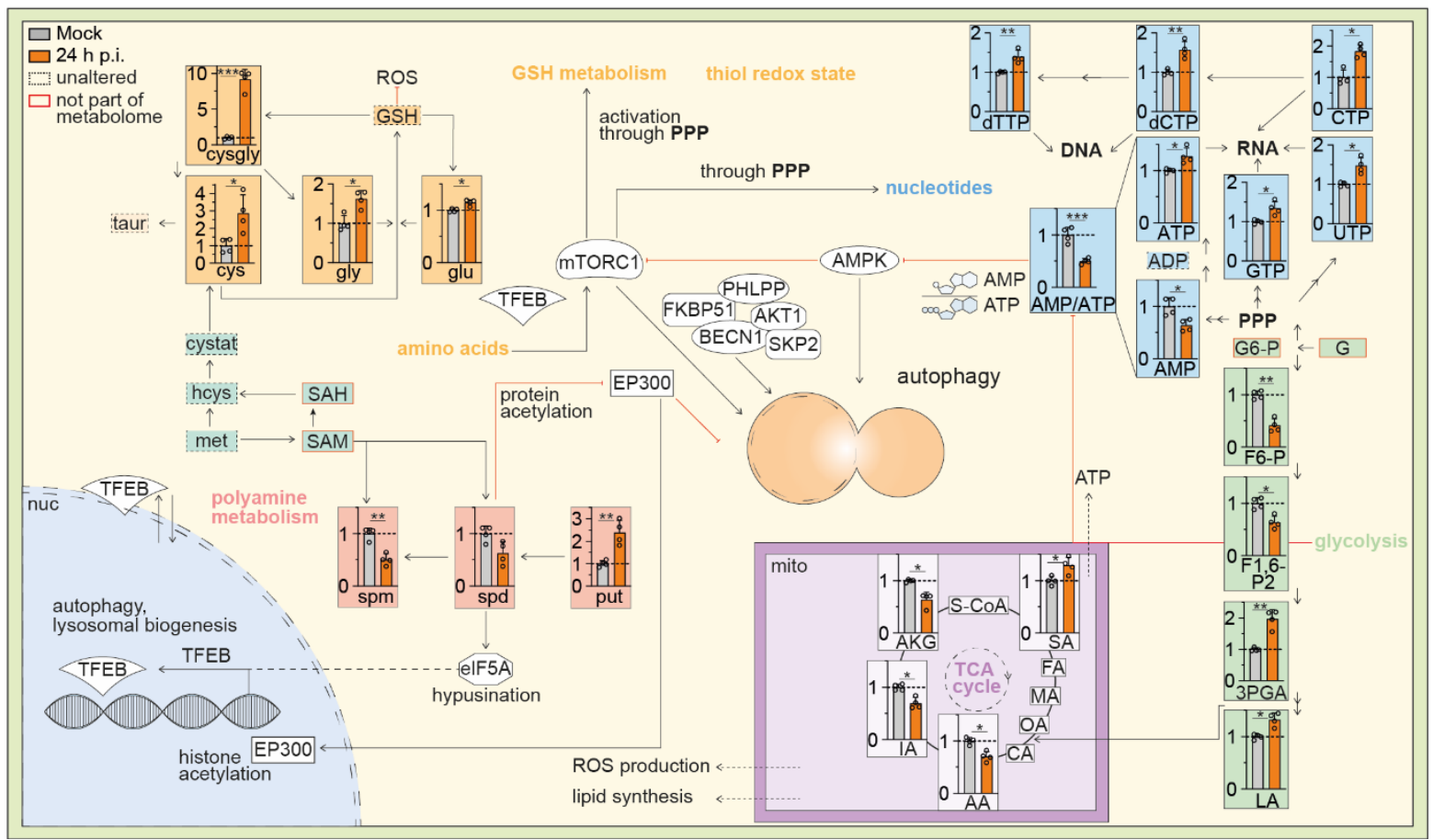

Fig.3: SARS-CoV-2 affects key metabolic pathways. (a) Analysis and regulation of significantly altered pathways of mock and SARS-CoV-2 infected ( $24 \mathrm{~h} \mathrm{p.i)} \mathrm{VeroFM} \mathrm{cells.} \mathrm{The} \mathrm{f}(\mathrm{x})$-axis shows the (median) log2 fold change (FC) of all significantly altered metabolites of the indicated pathway and the -log10 adjusted $p$-value (false discovery rate (FDR)) is shown on the $x$-axis. The size of the circles illustrates the number of significantly changed metabolites in relation to all metabolites of a specific pathway. $\mathrm{N}=4$ per group. (b) Volcano plot of metabolome of SARS-CoV-2 infected ( 24 h.p.i) VeroFM cells. Metabolites with $\log 2(F C) \geq 2$ and $-\log 10(F D R) \geq 1.3$ were considered significant. $N=4$ per group. (c) Analysis of the autophagic pathway and the involved metabolites: 'amino acids' and 'GSH metabolism' (orange), 'nucleotides' (blue), 'glycolysis/ TCA cycle' (violet) and 'polyamine metabolism' (red) and 'AMP/ATP ratio' (blue) upon mock and SARS-CoV-2 infected (24 h p.i) VeroFM cells. Error bars represent standard deviations *Adjusted $\mathrm{p}$-value $(\mathrm{FDR}) \leq 0.05 ;{ }^{*} \mathrm{FDR} \leq 0.01$; ***FD $\leq 0.001$. $\mathrm{N}=4$ per group. Abbreviations: taur, taurine; cysgly, cysteinylglycine; cys, cysteine; gly, glycine; glu, glutamate; cystat, cystathionine; hcys, homocysteine; met, methionine; SAH, S-adenosyl-L-homocysteine; SAM, S-adenosylmethionine; ROS, reactive oxygen species; GSH, glutathione; spm, spermine; spd, spermidine; put, putrescine; S-CoA, Succinyl-CoA; SA, succinic acid; FA, fumaric acid; MA, malic acid; CA, citric acid; AA, aconitic acid; IA, isocitric acid; AKG, $\alpha$-ketoglutaric acid; LA, lactic acid; 3PGA, 3phosphoglyceric acid; F1,6-P2, fructose 1,6-bisphosphate; F6-P, fructose 6-phosphate; G, glucose; G6-P, glucose 6-phosphate; AMP, adenosine monophosphate; ADP, adenosine diphosphate; ATP, adenosine triphosphate; GTP, guanosine triphosphate; UTP, uridine triphosphate; CTP, cytidine triphosphate; dCTP, deoxycytidine triphosphate; dTTP, deoxythymidine triphosphate; PPP, pentose phosphate pathway; TCA, tricarboxylic acid; FKBP51, 51 kDa FK506-binding protein; EP300, histone acetyltransferase $\mathrm{p300}$; TFEB, transcription factor EB; elF5A, eukaryotic translation initiation factor 5A; PHLPP, PH domain leucine-rich repeat-containing protein phosphatase; nuc, nucleus; mito, mitochondrion. 
a

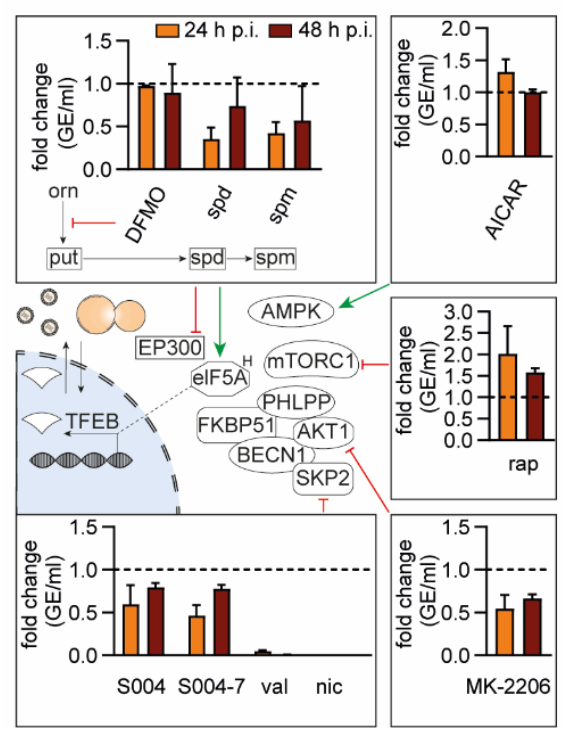

b

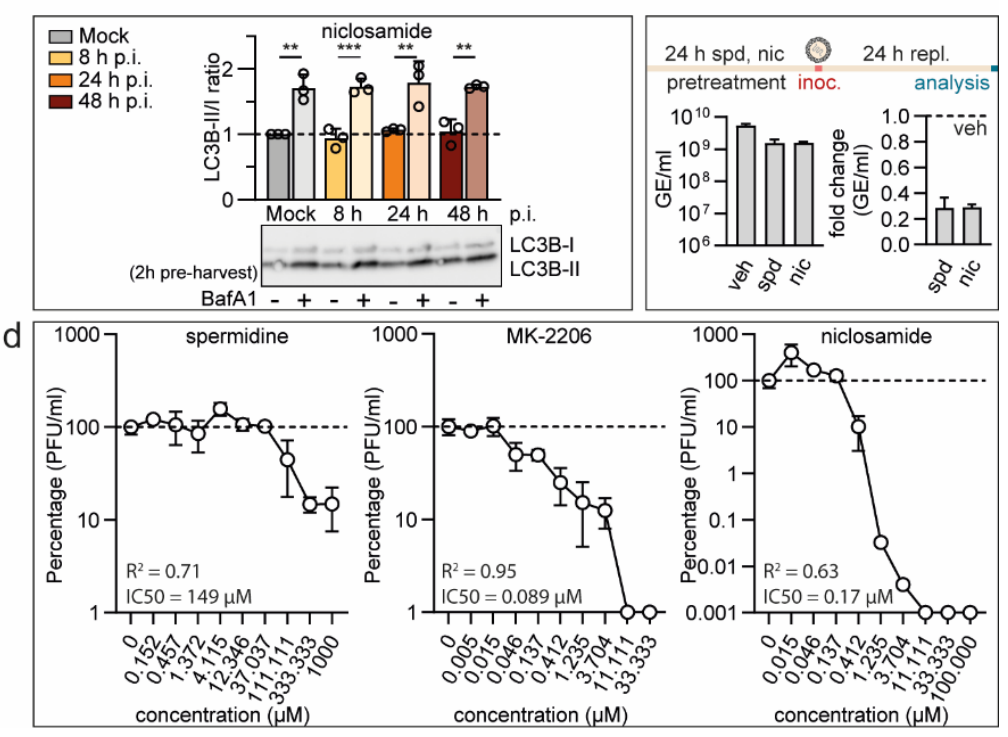

Fig.4: BECN1-stabilizing compounds and polyamines inhibit SARS-CoV-2 growth in cell cultures. (a) Schematic representation of autophagy signaling indicating site of action of small-molecule inhibitors used for pathway modulation, tested in SARS-CoV-2 growth assays. VeroFM cells were infected with SARS-CoV-2 (MOI $=0.0005$ ) and treated with (top, left) DFMO $(500 \mu \mathrm{M})$, spd (spermidine, $100 \mu \mathrm{M})$, spm (spermine, $100 \mu \mathrm{M})$ ), (top right) AICAR $(25 \mu \mathrm{M})$, (middle, right) rap (rapamycin, $300 \mathrm{nM}$ ), (bottom, left) SKP2 inhibitors S004 (SMIP004, $10 \mu \mathrm{M}$ ), S004-7 (SMIP004-7, $10 \mu \mathrm{M})$, val (valinomycin, 5 $\mu \mathrm{M})$, nic (niclosamide, $10 \mu \mathrm{M})$, (bottom, right) MK-2206 (1 $\mu \mathrm{M})$ or DMSO (vehicle, dashed lines). SARS-CoV-2 genome equivalents per $\mathrm{mL}$ (GE) were determined by real-time RT-PCR at $24 \mathrm{~h}$ and $48 \mathrm{~h}$ p.i., data are presented as fold difference. (b) Niclosamide treated $(10 \mu \mathrm{M})$ VeroFM cells were infected with SARS-CoV-2 (MOI $=0.0005)$ and incubated with bafilomycin A1 (BafA1, $100 \mathrm{nM}$ ) or vehicle (DMSO) for $2 \mathrm{~h}$ before samples were harvested at $8 \mathrm{~h}, 24 \mathrm{~h}$, or $48 \mathrm{~h}$ post infection (h p.i.). The ratios of LC3B-II/I were determined by Western blotting. (c) VeroFM cells were treated with spd (100 $\mu \mathrm{M})$, nic $(5 \mu \mathrm{M})$ or veh (vehicle) $24 \mathrm{~h}$ prior to infection with SARS-CoV-2 (MOI = 0.05). SARS-CoV-2 genome equivalents per $\mathrm{ml}(\mathrm{GE})$ were determined by real-time RT-PCR at $24 \mathrm{~h}$ p.i., data are presented $\mathrm{GE} / \mathrm{mL}$ (left) or as fold difference (right). (d) Concentration-dependent inhibition of SARS-CoV-2 growth by spermidine, MK-2206, and niclosamide. VeroFM cells were infected with SARS-CoV-2 $(\mathrm{MOI}=0.0005)$ and treated with different concentrations of spermidine, MK-2206, and niclosamide as shown in the figure. SARS-CoV-2 plaque forming units (PFU) were determined at $24 \mathrm{~h}$ (spermidine, MK-2206) and $48 \mathrm{~h}$ (niclosamide) p.i. by plaque assay. Data are presented as virus growth in percent. In all panels error bars denote standard error of mean derived from $n$ $=3$ biologically independent experiments. Abbreviations: orn, ornithine; DFMO, difluoromethylornithine; elF5AH, hypusinated elF5A; PFU, plaque-forming unit. 